\title{
Sec-mediated secretion by Coxiella burnetii
}

\author{
Christopher M Stead ${ }^{1}$, Anders Omsland ${ }^{2}$, Paul A Beare ${ }^{1}$, Kelsi M Sandoz ${ }^{1}$ and Robert A Heinzen ${ }^{1 *}$
}

\begin{abstract}
Background: Coxiella burnetii is a Gram-negative intracellular bacterial pathogen that replicates within a phagolysosome-like parasitophorous vacuole (PV) of macrophages. PV formation requires delivery of effector proteins directly into the host cell cytoplasm by a type IVB secretion system. However, additional secretion systems are likely responsible for modification of the PV lumen microenvironment that promote pathogen replication.

Results: To assess the potential of $C$. burnetii to secrete proteins into the PV, we analyzed the protein content of modified acidified citrate cysteine medium for the presence of C. burnetii proteins following axenic (host cell-free) growth. Mass spectrometry generated a list of 105 C. burnetii proteins that could be secreted. Based on bioinformatic analysis, 55 proteins were selected for further study by expressing them in C. burnetii with a C-terminal 3xFLAG-tag. Secretion of 27 proteins by C. burnetii transformants was confirmed by immunoblotting culture supernatants. Tagged proteins expressed by C. burnetii transformants were also found in the soluble fraction of infected Vero cells, indicating secretion occurs ex vivo. All secreted proteins contained a signal sequence, and deletion of this sequence from selected proteins abolished secretion. These data indicate protein secretion initially requires translocation across the inner-membrane into the periplasm via the activity of the Sec translocase.
\end{abstract}

Conclusions: C. burnetii secretes multiple proteins, in vitro and ex vivo, in a Sec-dependent manner. Possible roles for secreted proteins and secretion mechanisms are discussed.

Keywords: Coxiella, Secretion, Signal sequence, Outer membrane vesicles, Pili, Q fever, Sec-mediated

\section{Background}

Coxiella burnetii is a highly infectious Gram-negative intracellular bacterium that causes the zoonosis $Q$ fever [1]. Central to C. burnetii pathogenesis is the ability to proliferate within a parasitophorous vacuole (PV) of macrophages that has characteristics of a large phagolysosome $[2,3]$. By unknown mechanisms, the pathogen can resist the degradative activities of the vacuole while exploiting the biochemical and biophysical properties of the PV to promote robust intracellular replication $[4,5]$.

The $C$. burnetii PV is a unique cellular compartment that can occupy nearly the entire host cell cytoplasm [6]. C. burnetii protein synthesis is required for PV interactions with a subset of cellular vesicles that contribute material to the growing vacuole $[7,8]$. A collection of effector proteins secreted directly into the cytosol by a specialized

\footnotetext{
* Correspondence: rheinzen@niaid.nih.gov

${ }^{1}$ Coxiella Pathogenesis Section, Laboratory of Intracellular Parasites, Rocky Mountain Laboratories, National Institute of Allergy and Infectious Diseases, National Institutes of Health, Hamilton, MT 59840, USA

Full list of author information is available at the end of the article
}

Dot/Icm type IVB secretion system are considered largely responsible for modulation of host cell functions that promote PV formation [9-15], and dot/icm function is clearly necessary for productive infection $[9,10,16]$.

It is reasonable to suspect that modification of the PV microenvironment by additional secretion systems is also important in C. burnetii host cell parasitism. Gramnegative bacteria can employ several secretion systems to translocate proteins into the extracellular milieu [17]. However, bioinformatic analysis of the C. burnetii genome reveals canonical components of only a type I secretion system with the presence of a tolC homolog $[18,19]$. Type I secretion is typically a one step process that transports proteins directly from the bacterial cytoplasm into the surrounding environment [20]. However, a small number of proteins, such as heat-stable enterotoxins I and II of Escherichia coli [21,22], and an ankyrin repeat protein of Rickettsia typhi [23], appear to access TolC via the periplasm after transport across the inner membrane by the Sec translocase. C. burnetii lacks typical constituents of a type II secretion system [24]. However, the organism encodes several genes involved

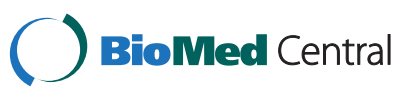


in type IV pili (T4P) assembly, several of which are homologous to counterparts of type II secretion systems, indicating a common evolutionary origin and possibly a similar function [25]. Accumulating data indicates core T4P proteins can constitute a secretion system [26-30]. In Francisella novicida, a collection of T4P proteins form a secretion system that secretes at least 7 proteins [27]. In Vibrio cholerae, T4P secrete a soluble colonization factor required for optimal intestinal colonization of infant mice [30]. Dichelobacter nodosus secrete proteases in a T4P-dependent manner $[29,31]$.

Like the well-studied type II secretion system of Legionella pneumophila, a close phylogenetic relative of C. burnetii [18], substrates secreted by T4P are biased towards $\mathrm{N}$-terminal signal sequence-containing enzymes [27,32]. $C$. burnetii encodes several enzymes with predicted signal sequences, such as an acid phosphatase (CBU0335) that inhibits neutrophil NADPH oxidase function and superoxide anion production $[33,34]$. Along with PV detoxification, $C$. burnetii exoenzymes could presumably degrade macromolecules into simpler substrates that could then be transported by the organism's numerous transporters [18]. Genome analysis indicates C. burnetii possesses a complete Sec translocase for translocation of signal sequencecontaining proteins into the periplasm $[18,19]$.

Another secretion mechanism employed by Gramnegative bacteria is release of outer membrane vesicles (OMVs). OMVs capture periplasmic components before the vesicle pinches off from the cell envelope. This 'packaging' of proteins is thought to provide a protective environment for delivery of the contents. OMVs are implicated in a variety of functions including delivery of virulence factors, killing of competing bacteria, and suppression of host immune responses [35,36].

The discovery of host cell-free growth of C. burnetii in acidified citrate cysteine medium (ACCM) [37,38] allowed us to probe culture media for the presence of secreted proteins. Mass spectrometry generated a list of 105 C. burnetii proteins in ACCM culture supernatants. Immunoblotting of culture supernatants following growth of C. burnetii transformants expressing individual epitope-tagged versions of identified proteins confirmed secretion of 27 of these proteins. Secretion of epitopetagged proteins also occurred during growth of $C$. burnetii in Vero host cells. An intact N-terminal signal sequence was required for secretion, indicating secreted proteins have a transient periplasmic location.

\section{Results}

\section{Coxiella burnetii proteins are present in growth medium supernatant}

The Dot/Icm type IVB secretion system of $C$. burnetii has been extensively studied [9,10,39]. However, little is known about other secretion systems of $C$. burnetii that are presumably important for intracellular parasitism. To determine if $C$. burnetii secretes proteins during axenic growth, bacteria were cultivated in ACCM-2 without neopeptone to eliminate media proteins. Following 7 days of growth, supernatant was concentrated and analyzed by SDS-PAGE and silver staining (Figure 1). Many proteins were detected, with the majority having a molecular weight below $20 \mathrm{kDa}$. In a discovery experiment to generate a list of potentially secreted proteins to further investigate, SDS-PAGE was conducted again and proteins stained with Coomassie G-250 to allow analysis by microcapillary reverse-phase HPLC nano-electrospray tandem mass spectrometry ( $\mu \mathrm{LC} / \mathrm{MS} / \mathrm{MS})$. A list of 105 proteins was generated (Additional files 1 and 2) with functions assigned based on the annotated genome of the C. burnetii Nine Mile RSA493 reference strain [18]. Sixteen proteins were annotated as hypothetical exported proteins, which represents $36 \%$ of the total proteins with this annotation in the predicted C. burnetii proteome [18]. Twenty-nine proteins, such as translation initiation factor 1 (InfA) and ribosomal protein subunit L31P (RpmE), were predicted as cytoplasmic using the PSORTb v3.0.2 bacterial protein subcellular localization prediction program [40]. This result could be explained by a small amount of bacterial lysis releasing abundant cytoplasmic proteins that are then detected by highly sensitive mass spectrometry. The only Dot/Icm type IVB secretion system substrate detected was CBU0937 [39]. However, type IVB-dependent secretion of CBU0937 was demonstrated using L. pneumophila as a surrogate host, and the protein contains a predicted signal sequence, which are typically not associated with Dot/Icm type IVB effectors [41]. Thus, CBU0937 may represent a false positive type IVB effector. Nonetheless, the lack of identified C. burnetii Dot/Icm type IVB secretion system substrates in culture supernatants indicates secretion via this mechanism requires host cell-derived signals.

\section{Expression of epitope-tagged proteins by C. burnetii transformants confirms secretion}

To confirm active secretion of proteins by $C$. burnetii into growth media, we generated 55 genetic transformants expressing individual proteins, under the control of an inducible TetA promoter, that contain a C-terminal 3xFLAG-tag (Additional file 2). Proteins identified by mass spectometry were selected for epitope-tagging based on predictions obtained using PSORTb, TMHMM [42], SignalP 3.0 [43], BLAST and PubMed bioinformatics tools. Each protein was first analyzed by a BLAST search to identify potential homologs. If a homolog was identified, PubMed searches were conducted to determine if the function and/or the cellular location of the homolog had been characterized. The predicted cellular location was also obtained using PSORTb, TMHMM 


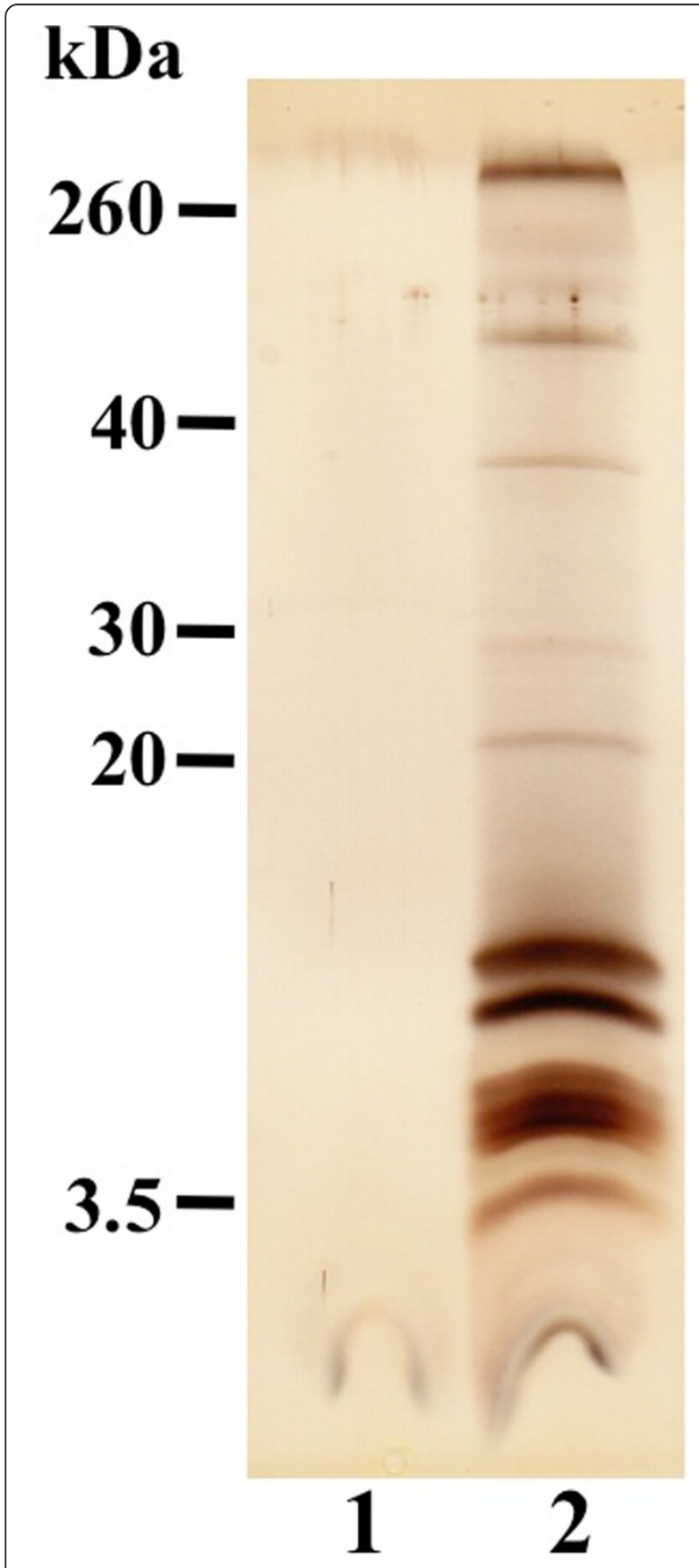

Figure 1 Multiple Coxiella burnetii proteins are present in growth medium supernatant. C. burnetii was cultured in modified ACCM-2 for 7 days, then supernatant was collected and analyzed by SDS-PAGE and silver staining. Lane 1, uninoculated media; lane 2, C. burnetii growth media.

and SignalP. Based on these analyses, proteins that were unlikely to be secreted, such as malate dehydrogenase, were eliminated from further study.

Expression of FLAG-tagged proteins by C. burnetii transformants was induced by addition of anhydrotetracycline (aTc) following $48 \mathrm{~h}$ of growth of individual transformants in ACCM-2. C. burnetii and culture supernatants were harvested $24 \mathrm{~h}$ later. Immunoblotting of culture supernatants with anti-FLAG antibody confirmed secretion of 27 of the 55 candidate proteins (Figure 2, Table 1 \& Additional file 3). FLAG-tag positive bands were not due to cell lysis as bands were not observed following probing of individual supernatants with antibody directed against EF-Ts, an abundant cytoplasmic protein (Figure 2 \& Additional file 3). To ensure negative secretion was not due to a lack of protein expression, bacterial pellets were also analyzed by immunoblotting using the anti-FLAG antibody. With the exception of CBU0089a, CBU1138, CBU1681, and CBU2027, expression of all tagged proteins was confirmed (Additional file 3).

All 27 secreted proteins contained a predicted signal peptide, with 19 annotated as hypothetical proteins (Table 1). This is not surprising given the unique hostpathogen relationship of $C$. burnetii and the fact that $40.3 \%$ of the open reading frames of the Nine Mile

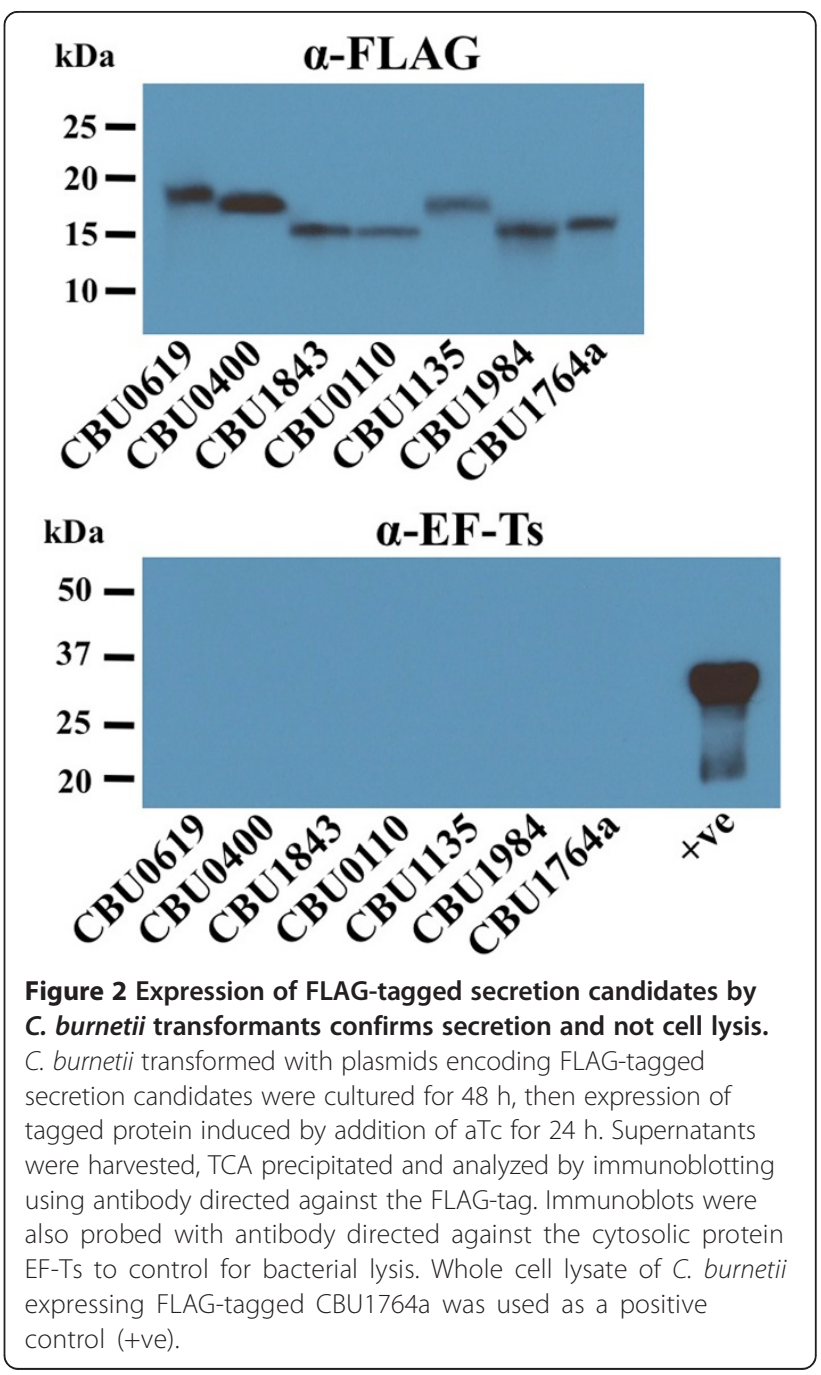


Table 1 Proteins identified in C. burnetii ACCM-2 culture supernatants by FLAG-tag assay

\begin{tabular}{lll}
\hline Protein & Annotation & kDa \\
\hline CBU0110 & Hypothetical exported protein & 13.0 \\
CBU0378 & Hypothetical membrane associated protein & 15.0 \\
CBU0400 & Hypothetical protein & 17.0 \\
CBU0482 & Arginine-binding protein (Artl) & 29.7 \\
CBU0562a & Hypothetical protein & 15.3 \\
CBU0619 & Hypothetical exported protein & 17.4 \\
CBU0630 & FKBP-type peptidyl-prolyl cis-trans isomerase (FkpA) & 25.5 \\
CBU0731 & Hypothetical exported protein & 15.4 \\
CBU0915 & Enhanced entry protein EnhB (EnhB1) & 19.4 \\
CBU0942 & Hypothetical exported protein & 14.0 \\
CBU1095 & Hypothetical exported protein & 17.9 \\
CBU1135 & Hypothetical exported protein & 15.9 \\
CBU1137 & Enhanced entry protein EnhB (EnhB2) & 20.9 \\
CBU1173 & Hypothetical protein & 13.7 \\
CBU1394 & Enhanced entry protein EnhA (EnhA5) & 19.4 \\
CBU1404 & Hypothetical exported protein & 12.3 \\
CBU1429a & Hypothetical protein & 12.6 \\
CBU1651 & Hypothetical membrane associated protein & 15.9 \\
CBU1764a & Hypothetical protein & 13.5 \\
CBU1822 & Superoxide dismutase [Cu-Zn] (SodC) & 17.9 \\
CBU1843 & Hypothetical exported protein & 14.7 \\
CBU1869 & Hypothetical exported protein & 24.8 \\
CBU1902 & Peptidase, M16 family & 52.0 \\
CBU1910 & Outer membrane protein (Com1) & 27.6 \\
CBU1930a & Hypothetical protein & 13.4 \\
CBU1984 & Hypothetical exported protein & 13.8 \\
CBU2072 & Hypothetical exported protein & \\
\hline & &
\end{tabular}

reference strain encode hypothetical proteins [18]. Secretion of proteins annotated as enhanced entry proteins (EnhB1, EnhB2 and EnhA5) was confirmed by the FLAGtag assay. These proteins are homologous to L. pneumophila proteins originally thought to facilitate pathogen entry into host cells (EnhA, B \& C) [44]. However, a more recent study of L. pneumophila EnhC demonstrates a role for this protein in peptidoglycan remodeling [45]. Secretion of Com1 and FkpA (Mip) was confirmed, both of which also have homologs in L. pneumophila. Little is known about their roles in C. burnetii pathogenesis, although Com 1 is known to be outer membrane associated [46] and FkpA has peptidyl-prolyl cis-trans isomerase (PPIase) activity [47]. The three remaining secreted proteins with predicted functions were ArtI (CBU0482), an arginine-binding protein, SodC (CBU1822), a $\mathrm{Cu}$ Zn superoxide dismutase, and a M16 family peptidase (CBU1902).

\section{C. burnetii secretes FLAG-tagged proteins during growth in host cells}

We next examined whether proteins secreted by $C$. burnetii during axenic growth were also secreted during growth in mammalian host cells. Vero cells were infected for 5 days with $C$. burnetii transformants expressing the FLAG-tagged secreted proteins CBU0110, CBU1135 or CBU1984. aTc was added to induce protein expression, then infected cells lysed $18 \mathrm{~h}$ later with $0.1 \%$ Triton X-100, which solubilizes host cell membranes, but not C. burnetii [13]. Cell lysates were centrifuged, then the pellets (containing C. burnetii and host cell debris) and supernatants were analyzed by immunoblotting using $\alpha$-FLAG and $\alpha$-EF-Ts antibodies (Figure 3). FLAG-tagged proteins were detected in the supernatant, indicating secretion occurs during host cell infection. FLAG-tagged proteins were also present in the bacterial pellet showing the rate of protein synthesis is greater than the rate of secretion. EF-Ts was only detected in the pellet, thereby eliminating bacterial lysis as a source of FLAG-tagged protein in supernatants.

\section{Secretion of FLAG-tagged proteins requires an intact signal sequence}

All verified secreted proteins contained a predicted Nterminal signal sequence. Signal sequences direct transport of proteins across the inner membrane via the Sec translocase [48]. To determine if transport to the periplasm was necessary for secretion, the secreted proteins CBU0110, CBU0915, CBU1135, CBU1173 and CBU1984

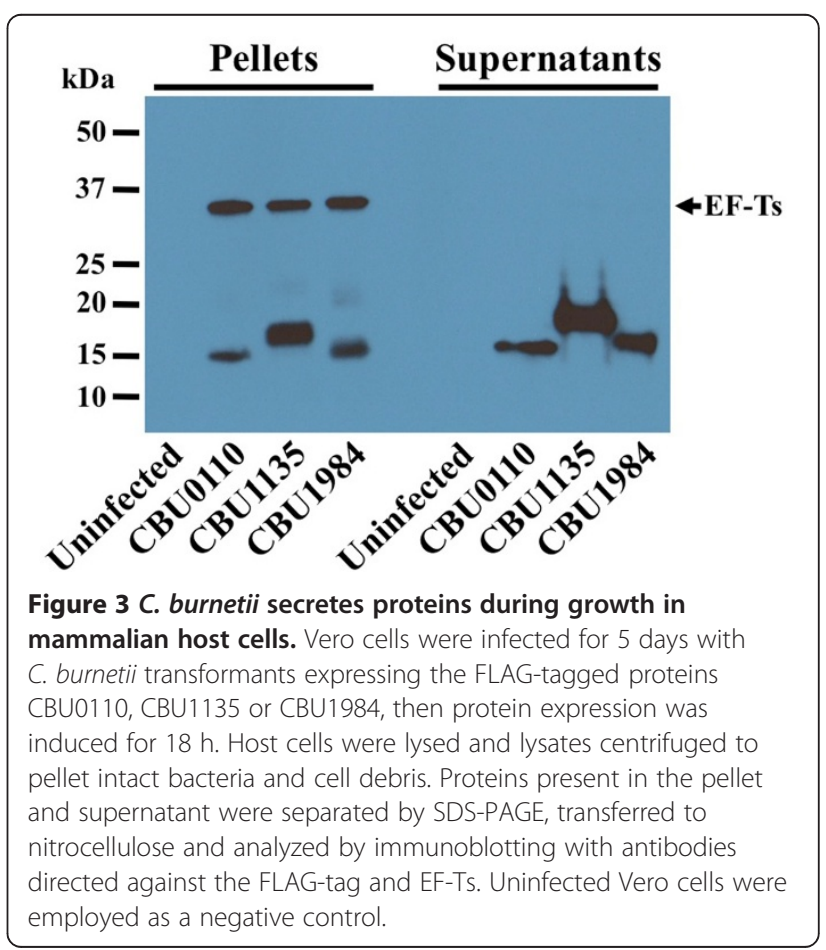


were expressed as before, but without their signal sequences. Immunoblotting for C-terminal FLAG-tags revealed that each of the five proteins was present in cell pellets, but not culture supernatants (Figure 4). Thus, a signal sequence, and therefore, a transient periplasmic location is necessary for secretion.

\section{Potential secretion mechanisms}

C. burnetii Sec-mediated secretion could occur by the mechanisms depicted in Figure 5. Type I-like secretion is predicted by the presence of a tolC homolog (CBU0056) in the C. burnetii genome. Genome analysis also makes T4P-mediated secretion conceivable as 13 T4P genes are present in the $C$. burnetii Nine Mile reference strain genome (Additional file 4). Eleven of these genes share homologs with the T4P genes of F. novicida, a bacterium that employs T4P-mediated secretion (Additional file 4). However, we did not detect pili on the surface of $C$. burnetii using a procedure that visualized pili on F. tularensis LVS [49] (Additional file 5). OMVs are produced by a large variety of microbes [50]. Figure 6 depicts what appear to be C. burnetii OMVs being produced by bacteria growing in media and within Vero cells, suggesting OMVs contribute to Sec-mediated secretion of proteins by C. burnetii.

\section{Discussion}

The importance of protein secretion for bacterial survival and virulence is well documented. Therefore, it was not surprising to discover that $C$. burnetii secretes at least 27 proteins into growth media. This number is similar to the 25 proteins experimentally confirmed by the laboratory of N. P. Cianciotto as secreted by the type II secretion system of $L$. pneumophila, a close relative of C. burnetii $[32,51]$. Heterogeneity among genes encoding secreted proteins is observed between the Nine Mile strain genome used in this study, and the published

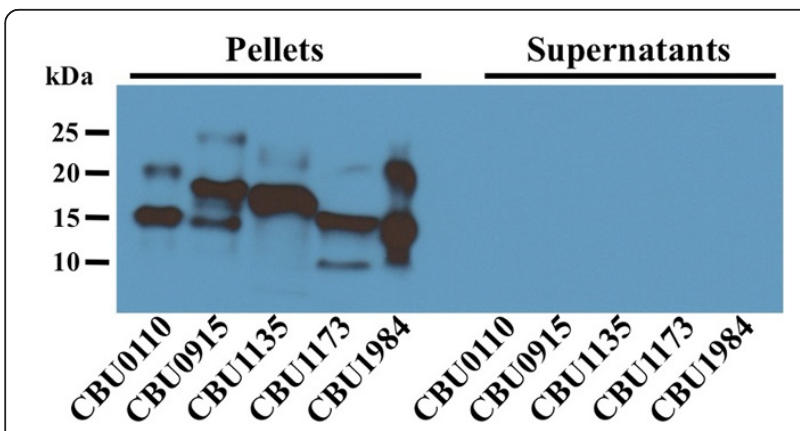

Figure 4 Secretion requires an intact signal sequence. Five secreted proteins (CBU0110, CBU0915, CBU1135, CBU1173 and CBU1984) without their respective signal sequence were expressed as described in Figure 2. Pellets and TCA precipitated supernatants were analyzed by immunoblotting using antibody directed against the FLAG-tag. genomes of the K (Q154), G (Q212), and Dugway (5J108-111) strains. Genes encoding CBU0400 and CBU0562a are missing in $\mathrm{K}$ and $\mathrm{G}$, respectively, and four genes are truncated as follows: CBU0110 and CBU1135 (G), CBU1429a (G and K), and CBU1822 (Dugway). All code for hypothetical proteins except CBU1822, which encodes SodC.

Assigning functional roles to these proteins is difficult given the majority are annotated as hypothetical proteins. However, recently developed methods for deleting C. burnetii genes could prove useful in defining function [16]. Of the few secreted proteins with predicted functions, SodC, ArtI, and an M16 family peptidase encoded by CBU1902, are of particular interest when considering the phagolysosomal characteristics of the C. burnetii PV. SodC is an important virulence factor of intracellular bacteria that degrades superoxide anion generated by the macrophage oxidative burst, thereby lowering oxidative stress [52]. The Dugway isolate may compensate for the lack of SodC by producing a functional catalase, which the Nile Mile strain apparently lacks [53]. ArtI might compensate for C. burnetii arginine auxotrophy [18] by high affinity binding of arginine in what might be a nutrient-limited PV environment. CBU1902 shares homology with Zn metalloendopeptidases, including pitrilysin, an E. coli peptidase that is capable of cleaving numerous substrates [54]. Thus, CBU1902 could modify the PV environment by cleaving harmful acid hydrolases or degrading complex proteinaceous material into peptides/amino acids suitable for transport by $C$. burnetii. Proteins secreted by Sec-mediated processes are clearly important for host cell modifications that benefit pathogen replication [24]. For example, Francisella spp. secrete an acid phosphatase (AcpA), both in vitro and ex vivo, that has been shown in macrophages to dephosphorylate components of the NADPH oxidase system. This suppression of the oxidative burst promotes intracellular survival and subsequent replication of the pathogen $[55,56]$. Interestingly, a similar scenario is invoked for the acid phosphatase of C. burnetii [34], although this protein was not among the 105 detected in growth media.

Based on genomic and/or ultrastructural data, we propose three secretion mechanisms/protein complexes that may contribute to Sec-mediated secretion by $C$. burnetii. First, the presence of several T4P genes organized in predicted operons suggests secretion might occur via a cell envelope-spanning complex comprised of T4P proteins. However, we found no evidence of pili-like structures on the surface of C. burnetii. To our knowledge, all bacteria that employ T4P-mediated secretion also produce identifiable T4P $[26,29,30]$. Furthermore, virulent C. burnetii strains display notable polymorphisms in pil gene composition. Specifically, pilN of the Nine Mile 


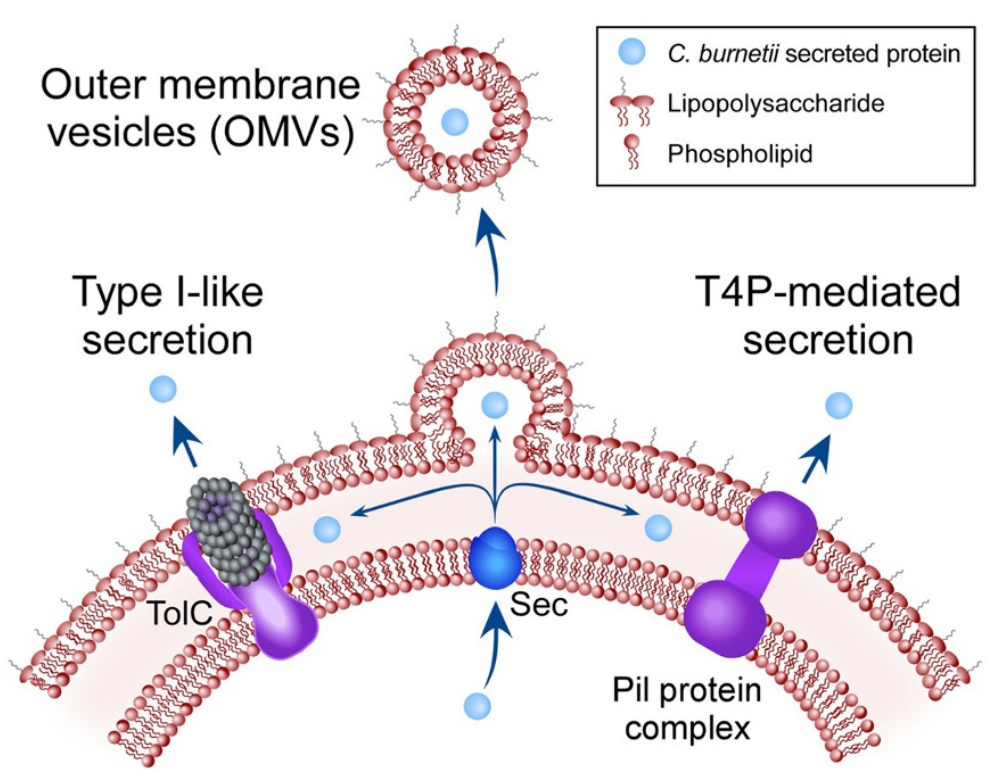

Figure 5 Possible Sec-mediated secretion mechanisms of $\boldsymbol{C}$. burnetii. Genome analysis predicts a TolC homolog that could mediate type I-like secretion from the periplasm after proteins are transported across the inner membrane by the Sec translocase. C. burnetii also encodes a set of core T4P proteins. T4P are evolutionarily related to type II secretion machinery and have been shown to mediate secretion of several proteins by F. novicida. Sequestration of periplasmic or surface proteins by OMVs is a third option for release of proteins into media supernatants.

strain, pilC of the $\mathrm{K}$ and $\mathrm{G}$ strains, and pilQ of the $\mathrm{G}$ and Dugway strains, are frameshifted and likely nonfunctional [18]. PilC and PilQ are necessary for secretion by F. novicida [27]. All strains also lack pilP, which is required for T4P production in several bacteria [57-60]. The incomplete and heterogeneous repertoire of $C$. burnetii T4P genes suggests the gene complement is undergoing genetic decay [18]. Second, secretion could occur by type I-like secretion. However, this process has been documented in relatively few bacteria and is usually responsible for secretion of a small number of proteins $[20,23]$. Thus, if type I-like secretion is employed by $C$. burnetii, it would likely be responsible for a small fraction of the secreted proteins. Third, and our favored hypothesis, is that the majority of proteins are secreted by OMVs. This idea is supported by EM showing
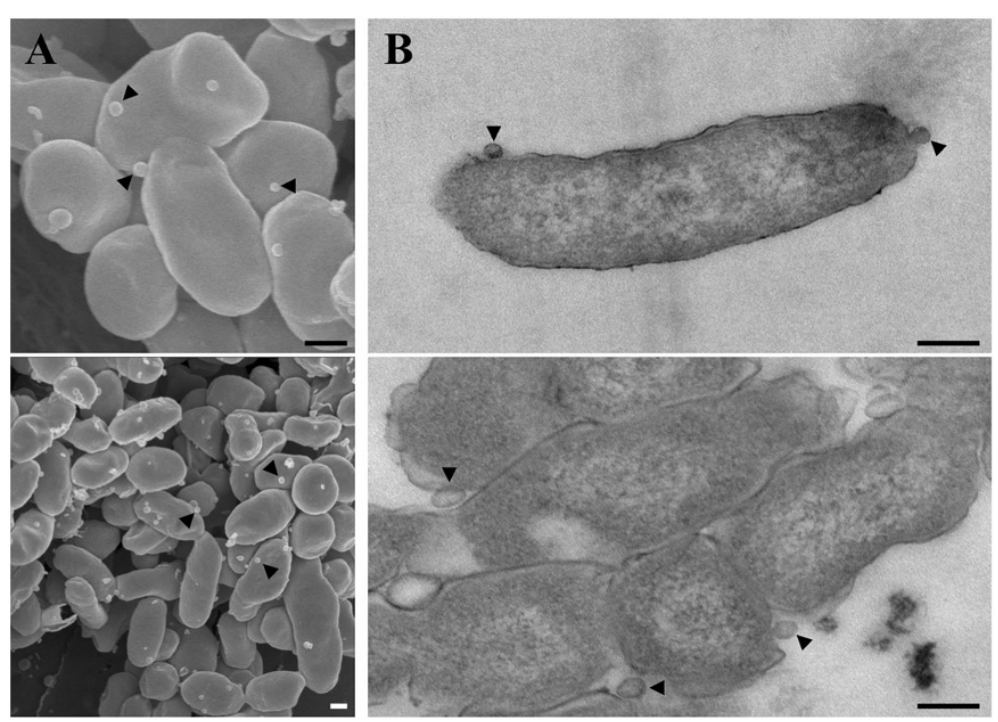

Figure 6 C. burnetii produces OMVs. (A) High and low magnification scanning electron micrographs of C. burnetii within the PV of infected Vero cells. Bacteria show membrane blebbing and OMVs (arrowheads). (B) Transmission electron micrographs of C. burnetii cultured in ACCM-2 for 2 days (upper panel) and 6 days (lower panel) showing membrane blebbing and OMVs (arrowheads). Scale bars $=0.2 \mu \mathrm{m}$. 
obvious membrane blebbing and OMV production during growth of $C$. burnetii in media and within mammalian host cells.

The possibility that $C$. burnetii proteins are secreted by OMVs is intriguing given the harsh environmental conditions of the PV lumen. The PV displays properties of a phagolysosome, such as acidic $\mathrm{pH}$ and active hydrolases, that can quickly degrade E. coli [3]. Sequestration of proteins by OMVs could provide a protective environment for delivery of virulence factors to targets within the PV and potentially to cytoplasmic targets should OMV contents transit the PV membrane. OMVs can also act as decoys by sequestering antimicrobial peptides before they reach their intended bacterial targets [61]. In the context of $C$. burnetii infection, it is tempting to speculate that, in addition to sequestering antimicrobial peptides, OMVs might detoxify superoxide by the activity of encapsulated SodC.

\section{Conclusions}

A list of 105 potentially secreted C. burnetii proteins was generated by mass spectrometry of culture supernatant. Twenty-seven of these proteins, from a pool of 55 candidate secreted proteins as determined bioinformatically, were confirmed to be secreted using C. burnetii transformants expressing FLAG-tagged versions and immunoblotting. Protein secretion was also detected ex vivo, suggesting that Sec-mediated secretion contributes to $C$. burnetii pathogenesis. All the secreted proteins had a signal sequence, which was verified as essential for secretion of 5 candidate proteins. Dependence on a signal sequence indicates that TolC, T4P or OMVs could mediate secretion.

\section{Methods}

\section{C. burnetii and mammalian cell lines}

C. burnetii Nine Mile phase II (RSA439, clone 4) was used in these studies [62]. For general bacterial culture, organisms were propagated microaerobically in ACCM$2+1 \%$ fetal bovine serum (FBS, Invitrogen) at $37^{\circ} \mathrm{C}$ [37] E. coli TOP10 (Invitrogen) or Stellar ${ }^{\mathrm{TM}}$ (BD Clontech) cells were used for recombinant DNA procedures and cultivated in Luria-Bertani (LB) broth. E. coli transformants were selected on LB agar plates containing $10 \mu \mathrm{g} / \mathrm{ml}$ of chloramphenicol. African green monkey kidney (Vero) cells (CCL-81; ATCC) were cultured using RPMI 1640 medium (Invitrogen) containing 10\% FBS (Invitrogen).

\section{SDS-PAGE and silver staining of $C$. burnetii culture supernatants}

Two $40 \mathrm{ml} \mathrm{C}$. burnetii cultures in ACCM-2 lacking neopeptone were grown in $125 \mathrm{ml}$ Erlenmyer flasks for 7 days with shaking at $75 \mathrm{rpm}$. The bacteria were combined and pelleted by centrifugation for $5 \mathrm{~min}$ at $20,000 \times g$, then the supernatant was passed through a $0.22 \mu \mathrm{m}$ syringe filter before being concentrated $\sim 400$ fold using a $3000 \mathrm{MWCO}$ centrifugal filter (Millipore). The concentrated supernatant was separated by SDSPAGE using a $16.5 \%$ gel and visualized by staining with the Silver Quest kit (Invitrogen).

\section{Microcapillary reverse-phase HPLC nano-electrospray tandem mass spectrometry ( $\mu \mathrm{LC} / \mathrm{MS} / \mathrm{MS}$ )}

Five $40 \mathrm{ml}$ C. burnetii cultures in ACCM-2 lacking neopeptone were grown in $125 \mathrm{ml}$ Erlenmyer flasks for 7 days with shaking at $75 \mathrm{rpm}$. The bacteria were combined and pelleted, then the supernatant passed through a $0.22 \mu \mathrm{m}$ syringe filter before being concentrated $\sim 500$ fold using a 3000 MWCO centrifugal filter. The concentrated supernatant was separated by SDS-PAGE using a $16.5 \%$ gel and visualized by staining with Coomassie G-250-based SimplyBlue SafeStain (Invitrogen). The protein containing lane was cut into 10 equal sections that were washed twice with 50\% acetonitrile, then stored at $-20^{\circ} \mathrm{C}$ prior to shipping to the Harvard Mass Spectrometry and Proteomics Resource Laboratory, FAS Center for Systems Biology, Northwest Bldg Room B247, 52 Oxford St, Cambridge MA. Gel sections were subjected to tryptic digestion and the resulting peptides sequenced by tandem mass spectrometry. Peptides were analyzed by microcapillary reverse-phase HPLC, directly coupled to the nano-electrospray ionization source of an LTQ-Orbitrap XL mass spectrometer (LCMSMS). Using a custom version of Proteomics Browser Suite (PBS; ThermoFisher Scientific), MS/MS spectra were searched against the $C$. burnetii subset of the NCBInr protein database concatenated to sequences of common laboratory contaminants. Methionine was allowed a variable modification for methionine sulfoxide and cysteine a fixed modification of carboxyamidomethyl cysteine. Peptide-spectrum matches were accepted with PBS filter sets to attain an estimated false discovery rate of $<1 \%$ using a decoy database strategy. Searches were performed with 2 missed cleavages, semi-tryptic, at 30 ppm mass tolerance, accepting only $+/-2.5 \mathrm{ppm}$. A minimum of 2 unique peptides were required to identify a protein.

\section{Construction of pJB-CAT-TetRA-3xFLAG}

The TetRA promoter/operator fragment was PCR amplified from pMiniTn7T-CAT::TetRA-icmDJB [9] using Accuprime $P f x$ (Invitrogen) and the primers TetRA-pJB$F$ and TetRA-3xFLAG-R obtained from Integrated DNA Technologies (Additional file 6). pJB-CAT-P1169-3xFLAG [63] was digested with EcoRI and PstI (New England Biolabs) to remove the P1169 promoter that was replaced with the TetRA fragment using the In-Fusion PCR cloning system (BD Clontech). 


\section{Construction of plasmids encoding C-terminal} FLAG-tagged proteins and transformation of $C$. burnetii Genes were PCR amplified with Accuprime Pfx and the primer sets listed in Additional file 6. SignalP 3.0 [43] was used to determine the location of signal sequences for the cloning of genes lacking this sequence. pJB-CAT-TetRA-3xFLAG was digested with PstI (New England Biolabs) followed by insertion of gene-encoding PCR products using the In-Fusion PCR cloning system (BD Clontech). C. burnetii was transformed with plasmid constructs as previously described [37].

\section{Immunoblotting of C. burnetii transformant culture supernatants}

Transformed C. burnetii expressing C-terminal 3xFLAGtagged proteins were cultivated in ACCM-2 + 1\% FBS for $48 \mathrm{~h}$, then expression of tagged proteins induced by addition of anhydrotetracycline (aTc, final concentration $=50 \mathrm{ng} / \mathrm{ml}$ ). Cell pellets and growth medium were collected $24 \mathrm{~h}$ after induction. One milliliter of supernatant from each sample was concentrated by trichloroacetic acid (TCA) precipitation (17\% final TCA concentration) prior to analysis by immunoblotting. Detection of proteins present in ACCM and/or the bacterial pellet was conducted by immunoblotting following separation of proteins by SDS-PAGE using a 4-20\% gradient gel (Pierce). Nitrocellulose membranes were incubated with monoclonal antibodies directed against FLAG (Sigma) or elongation factor Ts (EF-Ts; a generous gift of James Samuel, Texas A\&M University) [64]. Reacting proteins were detected using anti-mouse IgG secondary antibodies conjugated to horseradish peroxidase (Pierce) and chemiluminescence using ECL Pico or Femto reagent (Pierce).

\section{Ex vivo secretion assay}

The assay was performed essentially as described by Pan et al. [13]. Briefly, Vero cells cultured in 6-well tissue culture plates were infected for 5 days with $C$. burnetii expressing 3xFLAG-tagged proteins under the control of a TetA promoter. Protein expression was then induced with aTc (final concentration = $400 \mathrm{ng} / \mathrm{ml}$ ) for $18 \mathrm{~h}$. Cells were lysed with $0.1 \%$ Triton X-100 plus protease inhibitor cocktail (Sigma) in $1 \times$ phosphate buffered saline $\left(1.5 \mathrm{mM} \quad \mathrm{KH}_{2} \mathrm{PO}_{4}\right.$, $2.7 \mathrm{mM} \mathrm{Na} \mathrm{HPO}_{4}-7 \mathrm{H}_{2} \mathrm{O}, 155 \mathrm{mM} \mathrm{NaCl},[\mathrm{pH}$ 7.2]). Lysates were centrifuged for $10 \mathrm{~min}$ at $16,000 \times g$ and the supernatant passed through a $0.22 \mu \mathrm{M}$ syringe filter before TCA precipitation. Pellet and supernatant samples were separated by SDS-PAGE, transferred to nitrocellulose and probed with anti-FLAG and anti-EF-Ts antibodies.

\section{Transmission electron microscopy (EM) of C. burnetii grown in ACCM-2}

C. burnetii was grown in ACCM-2 for 2 or 6 days, then the cells were pelleted and fixed in $2.5 \%$ (vol/vol) glutaraldehyde with $0.05 \mathrm{M}$ sucrose in $0.1 \mathrm{M}$ sodium cacodylate buffer for $2 \mathrm{~h}$. Cells were post fixed in $0.5 \%$ reduced osmium using a Pelco Biowave microwave (Ted Pella) at $250 \mathrm{~W}$ under a 15-in $\mathrm{Hg}$ vacuum (all other chemical steps retained these settings) for $2 \mathrm{~min}$ on $/ 2 \mathrm{~min}$ off/ 2 min on. Next, tannic acid (1\%) was added and samples microwaved, followed by addition of 1\% uranyl acetate and microwaving. Samples were dehydrated in a graded ethanol series for $1 \mathrm{~min}$ under vacuum and infiltrated with 1:3, 1:1, and 3:1 (Epon/Araldite resin/ethanol), microwaved for $5 \mathrm{~min}$ on $/ 5 \mathrm{~min}$ off $/ 5 \mathrm{~min}$ on, then finally embedded in Epon/Araldite resin. Thin sections $(80 \mathrm{~nm})$ were cut using a Leica UC6 (Leica Microsystems) and sections stained with $1 \%$ uranyl acetate. Samples were viewed on a Hitachi $\mathrm{H}-7500$ transmission electron microscope (Hitachi) at $80 \mathrm{kV}$, and digital images were acquired with a Hamamatsu XR-100 digital camera system (AMT).

\section{Scanning EM of $C$. burnetii infected Vero cells}

Vero cells infected with C. burnetii for $48 \mathrm{~h}$ were fixed, postfixed, and dehydrated as described for transmission EM except that $1 \%$ reduced osmium was used for postfixation. Samples were then dried to the critical point in a Bal-Tec cpd 030 drier (Balzer). Cells were dryfractured by very lightly applying a small piece of adhesive tape to the apical surface that was subsequently gently removed. Cells were coated with $75 \AA$ of iridium in an IBS ion beam sputter (South Bay Technology). Samples were imaged on a Hitachi S-4500 scanning electron microscope (Hitachi).

\section{Transmission EM of negative stained C. burnetii and F. tularensis LVS}

A fixation and staining protocol optimized for preservation and visualization of pili was employed. F. tularensis subsp. holarctica Live Vaccine Strain (LVS) from a frozen stock was streaked onto a modified Mueller-Hinton plate that was incubated for $48 \mathrm{~h}$ at $37^{\circ} \mathrm{C}, 7 \% \mathrm{CO}_{2}$. Two milliliters of Chamberlain's defined medium was inoculated with $F$. tularensis LVS at $0.1 \mathrm{OD} / \mathrm{ml}$ and grown $\sim 16 \mathrm{~h}$ at $37^{\circ} \mathrm{C}, 200 \mathrm{rpm}$. The cells were pelleted, washed $2 \times$ with $1 \times$ PBS, then fixed with $4 \%$ paraformaldehyde (PFA). C. burnetii was cultured for 4 days in ACCM$2+1 \%$ FBS, the cells pelleted, washed $2 \times$ with $1 \times$ PBS, then fixed with $4 \%$ PFA. A $5 \mu$ l aliquot of fixed bacteria was allowed to settle on a formvar/carbon-coated grid for $5 \mathrm{~min}$. Liquid was removed with filter paper and the samples washed with $\mathrm{dH}_{2} \mathrm{O}$. Samples were stained with $2 \%$ ammonium molybdate for $2 \mathrm{~min}$. Remaining 
stain was removed with filter paper. Samples were viewed on a Hitachi H-7500 transmission electron microscope (Hitachi) at $80 \mathrm{kV}$, and digital images were acquired with a Hamamatsu XR-100 digital camera system (AMT).

\section{Availability of supporting data}

All supporting data are included as additional files.

\section{Additional files}

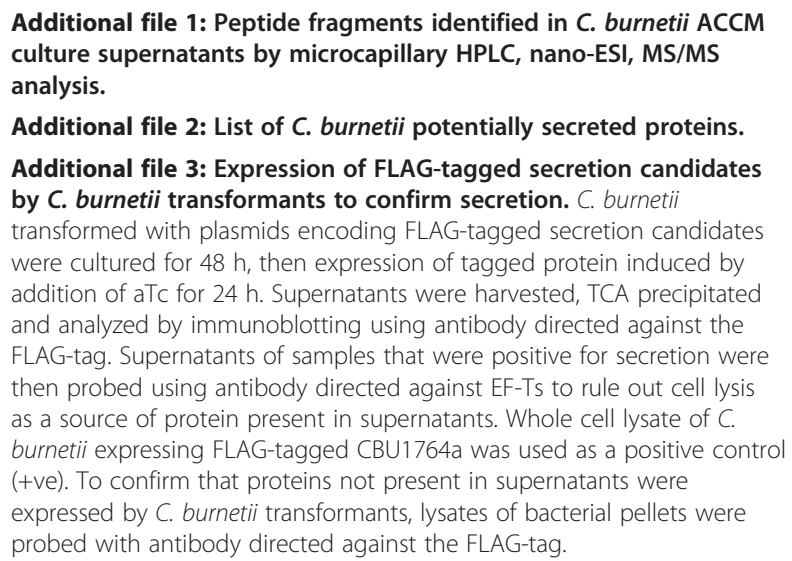

Additional file 4: Comparison of $F$. novicida and $C$. burnetii pil genes. The C. burnetii genome contains 13 pil genes, 11 of which are also present in the $F$. novicida genome, a bacterium that employs T4P-mediated secretion.

Additional file 5: $\boldsymbol{C}$. burnetii is not pilliated. Transmission electron micrographs of negatively stained bacteria show pili on F. tularensis LVS (panel A) but not C. burnetii (panel B). Scale bars $=0.5 \mu \mathrm{m}$.

Additional file 6: Primers used in this study.

\section{Competing interests}

The authors declare they have no competing interests.

\section{Authors' contributions}

CMS designed and conducted experiments and drafted the manuscript. AO conceived the study and conducted experiments. PAB constructed the expression vector and assisted with cloning. KMS carried out EM experiments. RAH participated in study design and coordination and helped to draft the manuscript. All authors read and approved the final manuscript.

\section{Acknowledgements}

We thank Elizabeth Fischer and Bryan Hansen of the Rocky Mountain Laboratories Microscopy Unit for electron microscopy, Jean Celli and Audrey Chong for Francisella samples, and Anita Mora and Austin Athman for graphic illustrations.

This work was supported by the Intramural Research Program of the National Institutes of Health, National Institute of Allergy and Infectious Diseases.

\section{Author details}

${ }^{1}$ Coxiella Pathogenesis Section, Laboratory of Intracellular Parasites, Rocky Mountain Laboratories, National Institute of Allergy and Infectious Diseases, National Institutes of Health, Hamilton, MT 59840, USA. ${ }^{2}$ Host-Parasite Interactions Section, Laboratory of Intracellular Parasites, Rocky Mountain Laboratories, National Institute of Allergy and Infectious Diseases, National Institutes of Health, Hamilton, MT 59840, USA.
Received: 3 July 2013 Accepted: 2 October 2013

Published: 5 October 2013

\section{References}

1. Maurin M, Raoult D: Q fever. Clin Microbiol Rev 1999, 12(4):518-553.

2. Voth DE, Heinzen RA: Lounging in a lysosome: the intracellular lifestyle of Coxiella burnetii. Cell Microbiol 2007, 9(4):829-840.

3. Howe D, Shannon JG, Winfree S, Dorward DW, Heinzen RA: Coxiella burnetii phase I and II variants replicate with similar kinetics in degradative phagolysosome-like compartments of human macrophages. Infect Immun 2010, 78(8):3465-3474.

4. Hackstadt T, Williams JC: Biochemical stratagem for obligate parasitism of eukaryotic cells by Coxiella burnetii. Proc Natl Acad Sci USA 1981, 78(5):3240-3244.

5. Omsland A, Heinzen RA: Life on the outside: the rescue of Coxiella burnetii from its host cell. Annu Rev Microbiol 2011, 65:111-128.

6. Coleman SA, Fischer ER, Howe D, Mead DJ, Heinzen RA: Temporal analysis of Coxiella burnetii morphological differentiation. J Bacteriol 2004, 186(21):7344-7352.

7. Gutierrez MG, Vazquez CL, Munafo DB, Zoppino FC, Beron W, Rabinovitch M, Colombo Ml: Autophagy induction favours the generation and maturation of the Coxiella-replicative vacuoles. Cell Microbiol 2005 7(7):981-993.

8. Howe D, Melnicakova J, Barak I, Heinzen RA: Maturation of the Coxiella burnetii parasitophorous vacuole requires bacterial protein synthesis but not replication. Cell Microbiol 2003, 5(7):469-480.

9. Beare PA, Gilk SD, Larson CL, Hill J, Stead CM, Omsland A, Cockrell DC, Howe D, Voth DE, Heinzen RA: Dot/Icm type IVB secretion system requirements for Coxiella burnetii growth in human macrophages. MBio 2011, 2(4):e00175-00111.

10. Carey KL, Newton HJ, Luhrmann A, Roy CR: The Coxiella burnetii Dot/lcm system delivers a unique repertoire of type IV effectors into host cells and is required for intracellular replication. PLoS Pathog 2011, 7(5):e1002056

11. Voth DE, Beare PA, Howe D, Sharma UM, Samoilis G, Cockrell DC, Omsland A, Heinzen RA: The Coxiella burnetii cryptic plasmid is enriched in genes encoding type IV secretion system substrates. J Bacterio/ 2011, 193(7):1493-1503.

12. Voth DE, Howe D, Beare PA, Vogel JP, Unsworth N Samuel JE, Heinzen RA The Coxiella burnetii ankyrin repeat domain-containing protein family is heterogeneous, with C-terminal truncations that influence Dot/lcmmediated secretion. J Bacteriol 2009, 191(13):4232-4242.

13. Pan $X$, Luhrmann A, Satoh A, Laskowski-Arce MA, Roy CR: Ankyrin repeat proteins comprise a diverse family of bacterial type IV effectors. Science 2008, 320(5883):1651-1654.

14. Luhrmann A, Nogueira CV, Carey KL, Roy CR: Inhibition of pathogeninduced apoptosis by a Coxiella burnetii type IV effector protein. Proc Natl Acad Sci USA 2010, 107(44):18997-19001.

15. Maturana P, Graham JG, Sharma UM, Voth DE: Refining the plasmidencoded Type IV secretion system substrate repertoire of Coxiella burnetii. J Bacteriol 2013, 195(14):3269-3276.

16. Beare PA, Larson CL, Gilk SD, Heinzen RA: Two systems for targeted gene deletion in Coxiella burnetii. Appl Environ Microbiol 2012, 78(13):4580-4589.

17. Tseng TT, Tyler BM, Setubal JC: Protein secretion systems in bacterial-host associations, and their description in the Gene Ontology. BMC Microbiol 2009, 9(Suppl 1):S2.

18. Beare PA, Unsworth N, Andoh M, Voth DE, Omsland A, Gilk SD, Williams KP, Sobral BW, Kupko JJ 3rd, Porcella SF, et al: Comparative genomics reveal extensive transposon-mediated genomic plasticity and diversity among potential effector proteins within the genus Coxiella. Infect Immun 2009, 77(2):642-656

19. Seshadri R, Paulsen IT, Eisen JA, Read TD, Nelson KE, Nelson WC, Ward NL, Tettelin H, Davidsen TM, Beanan MJ, et al: Complete genome sequence of the Q-fever pathogen Coxiella burnetii. Proc Natl Acad Sci USA 2003, 100(9):5455-5460.

20. Delepelaire P: Type I secretion in gram-negative bacteria. Biochim Biophys Acta 2004, 1694(1-3):149-161.

21. Foreman DT, Martinez Y, Coombs G, Torres A, Kupersztoch YM: TolC and DsbA are needed for the secretion of STB, a heat-stable enterotoxin of Escherichia coli. Mol Microbiol 1995, 18(2):237-245. 
22. Yamanaka H, Nomura T, Fujii Y, Okamoto K: Need for TolC, an Escherichia coli outer membrane protein, in the secretion of heat-stable enterotoxin I across the outer membrane. Microb Pathog 1998, 25(3):111-120.

23. Kaur SJ, Rahman MS, Ammerman NC, Beier-Sexton M, Ceraul SM, Gillespie J, Azad AF: TolC-dependent secretion of an ankyrin repeat-containing protein of Rickettsia typhi. J Bacteriol 2012, 194(18):4920-4932.

24. Cianciotto NP: Type II secretion: a protein secretion system for all seasons. Trends Microbiol 2005, 13(12):581-588.

25. Peabody CR, Chung YJ, Yen MR, Vidal-Ingigliardi D, Pugsley AP, Saier MH Jr: Type II protein secretion and its relationship to bacterial type IV pili and archaeal flagella. Microbiology 2003, 149(11):3051-3072.

26. Zogaj X, Chakraborty S, Liu J, Thanassi DG, Klose KE: Characterization of the Francisella tularensis subsp. novicida type IV pilus. Microbiology 2008, 154(7):2139-2150

27. Hager AJ, Bolton DL, Pelletier MR, Brittnacher MJ, Gallagher LA, Kaul R, Skerrett SJ, Miller SI, Guina T: Type IV pili-mediated secretion modulates Francisella virulence. Mol Microbiol 2006, 62(1):227-237.

28. Forsberg A, Guina T: Type II secretion and type IV pili of Francisella. Ann NY Acad Sci 2007, 1105:187-201.

29. Han X, Kennan RM, Parker D, Davies JK, Rood J: Type IV fimbrial biogenesis is required for protease secretion and natural transformation in Dichelobacter nodosus. J Bacteriol 2007, 189(14):5022-5033.

30. Kirn TJ, Bose N, Taylor RK: Secretion of a soluble colonization factor by the TCP type 4 pilus biogenesis pathway in Vibrio cholerae. Mol Microbiol 2003, 49(1):81-92.

31. Kennan RM, Dhungyel OP, Whittington RJ, Egerton JR, Rood Jl: The type IV fimbrial subunit gene ( imA) of Dichelobacter nodosus is essential for virulence, protease secretion, and natural competence. J Bacteriol 2001, 183(15):4451-4458

32. Debroy S, Dao J, Soderberg M, Rossier O, Cianciotto NP: Legionella pneumophila type II secretome reveals unique exoproteins and a chitinase that promotes bacterial persistence in the lung. Proc Natl Acad Sci USA 2006, 103(50):19146-19151.

33. Siemsen DW, Kirpotina LN, Jutila MA, Quinn MT: Inhibition of the human neutrophil NADPH oxidase by Coxiella burnetii. Microbes Infect 2009, 11(6-7):671-679.

34. Hill J, Samuel JE: Coxiella burnetii acid phosphatase inhibits the release of reactive oxygen intermediates in polymorphonuclear leukocytes. Infect Immun 2011, 79(1):414-420.

35. MacDonald IA, Kuehn MJ: Offense and defense: microbial membrane vesicles play both ways. Res Microbiol 2012, 163(9-10):607-618.

36. Mashburn-Warren LM, Whiteley M: Special delivery: vesicle trafficking in prokaryotes. Mol Microbiol 2006, 61(4):839-846.

37. Omsland A, Beare PA, Hill J, Cockrell DC, Howe D, Hansen B, Samuel JE, Heinzen RA: Isolation from animal tissue and genetic transformation of Coxiella burnetii are facilitated by an improved axenic growth medium. Appl Environ Microbiol 2011, 77(11):3720-3725.

38. Omsland A, Cockrell DC, Howe D, Fischer ER, Virtaneva K, Sturdevant DE, Porcella SF, Heinzen RA: Host cell-free growth of the Q fever bacterium Coxiella burnetii. Proc Natl Acad Sci USA 2009, 106(11):4430-4434.

39. Chen C, Banga S, Mertens K, Weber MM, Gorbaslieva I, Tan Y, Luo ZQ, Samuel JE: Large-scale identification and translocation of type IV secretion substrates by Coxiella burnetii. Proc Natl Acad Sci USA 2010, 107 (50):21755-21760

40. Yu NY, Wagner JR, Laird MR, Melli G, Rey S, Lo R, Dao P, Sahinalp SC, Ester $M$, Foster $\sqcup$, et al: PSORTb 3.0: improved protein subcellular localization prediction with refined localization subcategories and predictive capabilities for all prokaryotes. Bioinformatics 2010, 26(13):1608-1615.

41. Alvarez-Martinez CE, Christie PJ: Biological diversity of prokaryotic type IV secretion systems. Microbiol Mol Biol Rev 2009, 73(4):775-808.

42. Krogh A, Larsson B, von Heijne G, Sonnhammer EL: Predicting transmembrane protein topology with a hidden Markov model: application to complete genomes. J Mol Biol 2001, 305(3):567-580

43. Bendtsen JD, Nielsen H, von Heijne G, Brunak S: Improved prediction of signal peptides: SignalP 3.0. J Mol Biol 2004, 340(4):783-795.

44. Cirillo SL, Lum J, Cirillo JD: Identification of novel loci involved in entry by Legionella pneumophila. Microbiology 2000, 146(6):1345-1359.

45. Liu M, Haenssler E, Uehara T, Losick VP, Park JT, Isberg RR: The Legionella pneumophila EnhC protein interferes with immunostimulatory muramyl peptide production to evade innate immunity. Cell Host Microbe 2012, 12(2):166-176.
46. Hendrix LR, Mallavia LP, Samuel JE: Cloning and sequencing of Coxiella burnetii outer membrane protein gene com1. Infect Immun 1993, 61(2):470-477

47. Mo YY, Cianciotto NP, Mallavia LP: Molecular cloning of a Coxiella burneti gene encoding a macrophage infectivity potentiator (Mip) analogue. Microbiology 1995, 141(11):2861-2871.

48. du Plessis DJ, Nouwen N, Driessen AJ: The Sec translocase. Biochim Biophys Acta 2011, 1808(3):851-865.

49. Chakraborty S, Monfett M, Maier TM, Benach JL, Frank DW, Thanassi DG: Type IV pili in Francisella tularensis: roles of pilF and pilT in fiber assembly, host cell adherence, and virulence. Infect Immun 2008, 76(7):2852-2861

50. Deatherage BL, Cookson BT: Membrane vesicle release in bacteria, eukaryotes, and archaea: a conserved yet underappreciated aspect of microbial life. Infect Immun 2012, 80(6):1948-1957.

51. Cianciotto NP: Many substrates and functions of type II secretion: lessons learned from Legionella pneumophila. Future Microbiol 2009, 4(7):797-805.

52. Battistoni $\mathrm{A}$ : Role of prokaryotic $\mathrm{Cu}, \mathrm{Zn}$ superoxide dismutase in pathogenesis. Biochem Soc Trans 2003, 31(6):1326-1329.

53. Mertens K, Samuel JE: Defense mechanisms against oxidative stress in Coxiella burnetii: adaptation to a unique intracellular niche. Adv Exp Med Biol 2012, 984:39-63.

54. Cornista J, Ikeuchi S, Haruki M, Kohara A, Takano K, Morikawa M, Kanaya S: Cleavage of various peptides with pitrilysin from Escherichia coli: kinetic analyses using beta-endorphin and its derivatives. Biosci Biotechnol Biochem 2004, 68(10):2128-2137.

55. Dai S, Mohapatra NP, Schlesinger LS, Gunn JS: The acid phosphatase AcpA is secreted in vitro and in macrophages by Francisella spp. Infect Immun 2012, 80(3):1088-1097.

56. Mohapatra NP, Soni S, Rajaram MV, Dang PM, Reilly TJ, El-Benna J, Clay CD, Schlesinger LS, Gunn JS: Francisella acid phosphatases inactivate the NADPH oxidase in human phagocytes. J Immunol 2010, 184(9):5141-5150.

57. Carbonnelle E, Helaine S, Prouvensier L, Nassif $X$, Pelicic V: Type IV pilus biogenesis in Neisseria meningitidis: PilW is involved in a step occurring after pilus assembly, essential for fibre stability and function. Mol Microbiol 2005, 55(1):54-64.

58. Martin PR, Watson AA, McCaul TF, Mattick JS: Characterization of a five-gene cluster required for the biogenesis of type 4 fimbriae in Pseudomonas aeruginosa. Mol Microbiol 1995, 16(3):497-508.

59. Nudleman E, Wall D, Kaiser D: Polar assembly of the type IV pilus secretin in Myxococcus xanthus. Mol Microbiol 2006, 60(1):16-29.

60. Roine E, Nunn DN, Paulin L, Romantschuk M: Characterization of genes required for pilus expression in Pseudomonas syringae pathovar phaseolicola. J Bacterio/ 1996, 178(2):410-417.

61. Manning AJ, Kuehn MJ: Contribution of bacterial outer membrane vesicles to innate bacterial defense. BMC Microbio/ 2011, 11:258.

62. Beare PA, Samuel JE, Howe D, Virtaneva K, Porcella SF, Heinzen RA: Genetic diversity of the $\mathrm{Q}$ fever agent, Coxiella burnetii, assessed by microarraybased whole-genome comparisons. J Bacterio/ 2006, 188(7):2309-2324.

63. Beare PA: Genetic manipulation of Coxiella burnetii. Adv Exp Med Biol 2012, 984:249-271.

64. Seshadri R, Hendrix LR, Samuel JE: Differential expression of translational elements by life cycle variants of Coxiella burnetii. Infect Immun 1999, 67(11):6026-6033.

doi:10.1186/1471-2180-13-222

Cite this article as: Stead et al: Sec-mediated secretion by Coxiella burnetii. BMC Microbiology 2013 13:222. 